\title{
Configurações
}

Revista Ciências Sociais

$10 \mid 2012$

Políticas Públicas

\section{O processo de construção da política de saúde do trabalhador no Brasil para o setor público}

The Formulation Process of the Worker Health Policy in Brazil for the public setor

Le processus de construction de la politique de santé au travail au Brésil pour le secteur public

Elsa Thomé de Andrade, Maria Inês Carsalade Martins e Jorge Huet Machado

\section{OpenEdition}

\section{Journals}

Edição electrónica

URL: http://journals.openedition.org/configuracoes/1472

DOI: $10.4000 /$ configuracoes. 1472

ISSN: 2182-7419

\section{Editora}

Centro de Investigação em Ciências Sociais

\section{Edição impressa}

Data de publição: 1 dezembro 2012

Paginação: 137-150

ISBN: 1646-5075

ISSN: 1646-5075

\section{Refêrencia eletrónica}

Elsa Thomé de Andrade, Maria Inês Carsalade Martins e Jorge Huet Machado, « O processo de construção da política de saúde do trabalhador no Brasil para o setor público », Configurações [Online], 10 | 2012, posto online no dia 19 setembro 2014, consultado o 10 dezembro 2020. URL : http:// journals.openedition.org/configuracoes/1472; DOI : https://doi.org/10.4000/configuracoes. 1472 


\section{O processo de construção da política de saúde do trabalhador no Brasil para o setor público}

The Formulation Process of the Worker Health Policy in Brazil for the public setor

Le processus de construction de la politique de santé au travail au Brésil pour le secteur public

Elsa Thomé de Andrade, Maria Inês Carsalade Martins e Jorge Huet Machado

\section{Introdução}

1 Considerando que as políticas sociais estão voltadas para a reprodução dos indivíduos e das coletividades, compreender as políticas de saúde como uma política social implica em reconhecer saúde como direito de cidadania, o que inclui, fundamentalmente, participação política e proteção social( Fleury, 2009). Assim, da mesma forma que as políticas públicas em seu conjunto, as políticas de saúde são construídas a partir de uma disputa que envolve necessidades de saúde, escolhas da comunidade, decisões políticas e recursos disponíveis.

2 A Organização Mundial de Saúde define a política de saúde como um “posicionamento ou procedimento estabelecido por instituições oficiais competentes, notadamente governamentais, que definem as prioridades e os parâmetros de ação em resposta às necessidades de saúde, aos recursos disponíveis e a outras pressões políticas..." (WHO, 1998:10).

3 Ao discutir a Política de Saúde, na perspetiva de uma Política Pública, Fleury (2009) destaca a importância da mesma como instrumento que evidencia a intencionalidade, objetivos e estratégias dos governos, através do qual se estabelece um compromisso com a população. “O cerne da política é constituído pelo seu propósito, diretrizes e definição de 
responsabilidades das esferas de governo e dos órgãos envolvidos" (Brasil, 1998:7 apud Fleury, 2009:41). O processo histórico de construção de uma política de saúde, portanto, envolve uma relação entre Estado, sociedade e mercado e expressa os diferentes momentos políticos, econômicos e sociais através das agendas públicas e do papel assumido pelo Estado, pela iniciativa privada e pela sociedade civil.

Através de um ensaio crítico, neste artigo procuraremos discutir o processo de construção da Política de Saúde para os trabalhadores do setor público no Brasil. O percurso metodológico realizado para analisar o processo de implementação da Política de Atenção à Saúde e Segurança do Servidores Públicos - (PASS) e sua base legal, busca evidenciar a distância entre o discurso e as propostas, a partir da compreensão do modo de inserção destas últimas na conjuntura dos contextos sociais, considerando os fatores políticos, econômicos e ideológicos nos quais se insere.

O ponto de partida, então, para a realização desta reflexão foi o acompanhamento das iniciativas de implementação deste novo sistema que traduz uma política específica de atenção à saúde para o servidor público e que deverá regular as ações vinculadas às relações de trabalho e saúde nas instituições públicas federais. Assim, é utilizado como modelo teórico a investigação qualitativa, considerando a sua preocupação de interpretação da ação social, focalizando o trabalho de grupos no processo, a nível regional e nacional, buscando entender não só a forma de intervenção dos trabalhadores do setor público federal, mas também a interpretação que dão a suas experiências.

Para o desenvolvimento do trabalho foi considerado de que o grau de participação dos gestores e trabalhadores e suas conceções sobre a relação saúde e trabalho e seus determinantes vão configurar diferentes modelos de atenção e organização dos serviços que se aproximam ou não do modelo de saúde integral.

7 Partindo da premissa de que o campo de formulação e implementação de políticas públicas é um espaço de lutas, política e ideológica, que se refletem nas agendas públicas e na relação do Estado com a sociedade serão utilizados como referência os conceitos de políticas sociais e de saúde (Fleury,2009); saúde do trabalhador (Oddone,1986); a importância da participação dos trabalhadores na gestão de seu processo de trabalho, com impactos diretos no processo saúde doença e os principais conceitos de trabalho, de saúde e de cidadania (Laurel e Noriega,1989); regulação do trabalho (Dedecca, 2006); e avaliação de políticas de saúde (Viana e Batista,2009).

Diante dessas bases teóricas e das observações empíricas do processo de implementação e definição de propostas do SIASS é realizada uma análise dos documentos produzidos até então nesse âmbito, os documentos oficiais e a legislação que institui a Política Nacional de Segurança e Saúde do Trabalhador (PNSST) e a Política de Atenção à Saúde dos Servidores públicos (PASS), à luz das abordagens trazidas por estes autores.

\section{Saúde e Trabalho no contexto das políticas públicas}

9 A progressiva inter-relação, entre ciência, tecnologia e produção de bens e serviços, que se acentua na segunda metade do século XX, vem transformando o modo de produção capitalista, introduzindo novas formas de relação e, com isto, a saúde passa a ser objeto de planejamento e de políticas dos governos numa perspetiva de retração do Estado e de expansão do mercado. 

enfraquecem o poder de reivindicação coletiva dos trabalhadores e reduz o papel do Estado nas relações de trabalho, o que diminui a regulação e, consequentemente, restringe também a proteção social.

11 Há uma mudança de relações entre sociedade e Estado e nos avanços conquistados no Welfare State com relação ao fortalecimento do atendimento das demandas da sociedade. 0 Estado não é mais entendido como intermediador e provedor sendo o pensamento hegemônico o de que os mercados devem funcionar livremente, tendo o Estado apenas o papel de regulador. Em nível político vamos ter o ideário neoliberal liderando e organizando as estruturas estatais, com diferentes rítmos e formas em cada nação. (Gomes, 2006)

12 Paralelamente e na contra mão das mudanças que vinham acontecendo no capitalismo e seus impactos no mundo do trabalho, a experiência do movimento operário italiano, nos anos 1960, recolocou a centralidade do trabalhador no processo de trabalho e contribuiu para a construção de um novo conceito para a saúde no trabalho. A Saúde do Trabalhador passa a ser considerada em sua dimensão política, sendo a busca pela transformação da realidade do trabalho a base do modelo de intervenção. Para Tambellini (1988), o modelo de saúde do trabalhador, que tem origem no movimento operário italiano “(...) é a expressão do poder dos trabalhadores de ter saúde e tomar em suas mãos o controle de suas próprias vidas, saúde e trabalho" (Tambellini, 1988: 12).

Esta experiência do Movimento Operário Italiano tem sido a base conceitual para os movimentos sociais e acadêmicos no sentido de construir um novo conceito para a saúde no trabalho, colocando o trabalhador como agente de produção de saúde, a partir da transformação do seu processo de trabalho.

14 De acordo com Oddone (1986), o Movimento Operário Italiano mostra a realidade com base nos saberes formais e informais da experiência individual validada pelo grupo de trabalhadores. Esta forma de investigação vem acompanhada pela criação do conceito de Comunidade Científica Ampliada e, assim, há compreensão da importância do encontro dos trabalhadores com pesquisadores num movimento de construção do saber e poder de intervenção nos locais de trabalho. Trata-se de um diálogo crítico entre os saberes fundados na experiência tendo como protagonista o "grupo operário homogêneo", com seu saber "informal", e o conhecimento científico, com o saber "formal". Oddone (1986: 77) afirma que:

“(...) o poder de ação dos coletivos dos trabalhadores (...) a tarefa consiste em inventar ou reinventar os instrumentos dessa ação, não mais iniciando protesto contra pressões ou "as negociando", mas pela via de sua superação concreta." (Oddone, 1986:77)

O princípio da saúde integral do trabalhador invoca o direito à saúde no seu sentido irrestrito da cidadania plena. Na prática, se traduz em ações que incluem a promoção, a prevenção e a assistência, a serem executadas de forma integrada e sempre com o objetivo do alcance da saúde integral do trabalhador, através de uma abordagem interdisciplinar e intersetorial. Inclui-se, assim, uma ação de articulação com ampla e efetiva participação de trabalhadores, de técnicos e pesquisadores, de instituições de ensino, de representantes sindicais, de serviços, da sociedade civil organizada e de outros atores institucionais e sociais. É importante que, dessa forma, seja forjada uma aliança tácita, no sentido de conseguir do Estado uma política coerente com as reais demandas.

Configurações, 10 | 2012 
16 Segundo esta conceção, a participação do trabalhador no processo de construção de políticas é o que legitima toda a eficácia de qualquer política pública. Este ator detém o conhecimento ou, podemos dizer, o saber do processo de trabalho e do impacto do trabalho em sua saúde e, portanto em sua vida.

17 O campo da Saúde do Trabalhador torna-se uma prática social instituinte, podendo contribuir para a transformação da realidade da saúde não só dos trabalhadores, mas da população como um todo, a partir da compreensão dos processos de trabalho particulares, de forma articulada com o consumo de bens e serviços e com o conjunto de valores, crenças, ideias e representações sociais próprias deste momento da história humana (Dias,1994).

18 A natureza participativa presente na Política de Saúde do Trabalhador para o setor público no Brasil contrasta com a visão de que a implantação de serviços de saúde e segurança no trabalho no âmbito das empresas está calcada em objetivos de promoção da saúde para redução do custo e em mudanças no comportamento individual (Alexander, 1988; Conrad \& Walsh, 1992).

\section{A regulação do Trabalho e a Proteção Social no Brasil}

19 No Brasil, a legislação trabalhista, com o objetivo de proteção e manutenção da saúde do trabalhador, ocorre de forma tardia em relação aos países desenvolvidos.

20 País de desenvolvimento e industrialização retardatária, o Brasil não seguiu a mesma trajetória de desenvolvimento industrial dos países da Europa e dos EUA. No período em que estes países iniciavam um processo de regulação do mercado de trabalho, estávamos ainda saindo de uma sociedade escravocrata, com uma economia fortemente agrícola até a década de 1950 .

21 O processo de industrialização no Brasil se iniciou na década de 30, num contexto de um governo de base ditatorial, que procurava consolidar o Estado Nacional estimulando o processo de industrialização nacional e ampliando a participação do Estado nas diversas esferas administrativas. É neste cenário que se introduz alguma regulação sobre o contrato de trabalho, ainda que mantendo uma relação assimétrica entre capital e trabalho, e se implementa uma política pública, embora pouco integrada e ainda de baixa cobertura no mercado de trabalho. (Dedecca, 2006).

Pressionado pelas influências das transformações acontecidas na Europa em relação às normas de proteção ao trabalhador, a adesão do Brasil em participar como signatário da OIT e com os movimentos operários aumentados com a emergência da industrialização, o governo brasileiro inicia assim seu processo de regulação do trabalho. Em 1923 é promulgada a primeira lei base da previdência social. Na sequência, em 1930, é criado o Ministério do Trabalho e em 1943 a Consolidação das Leis do Trabalho (CLT) com o objetivo de reunir as leis existentes, estabelecendo normas para regular as relações individuais e coletivas de trabalho. Esta é a primeira lei geral que se aplica a todos os empregados, sem distinção da natureza do trabalho e que, diante da dinâmica da vida no trabalho sofre alterações até aos dias de hoje. 
que passou a ser conhecido como cidadania regulada. Atualmente as leis trabalhistas se estendem a todos os trabalhadores com contrato formal de trabalho.

O sindicalismo, no Brasil, historicamente teve avanços e retrocessos, evidenciado em suas experiências organizativas, com maior maior ou menor conflito em lutas para sua regulamentação. A Constituição de 1988 assegurou aos servidores públicos o direito à livre associação sindical e o direito de greve, mas não há regulamentação do direito de greve e negociação coletiva.

No período de 1950 a 1964 com a democratização e o crescimento do país se inicia um processo de democratização da regulação do contrato de trabalho, ampliando-se o sistema de proteção social e ao trabalho. Este processo é interrompido com a instauração de um novo regime autoritário em 1964, que mantém a natureza formal da regulação pública. A democracia se reinstaura no país, em 1985, com a queda do governo militar e a convocação de uma Assembleia Nacional Constituinte.

A nova Constituição promulgada em 1988 representa uma conquista política e um avanço no sentido da democratização, da equidade e da cidadania, sendo conhecida como a "Constituição Cidadã". Nela instituiu-se entre outras conquistas sociais a universalização da saúde através do Sistema Único de Saúde (SUS) e um novo arcabouço jurídicoinstitucional para o serviço público, o Regime Jurídico Único(RJU) o qual normatiza as contratações no setor público. O RJU determina a necessidade de submeter o servidor público a um regime próprio, com ingresso nas carreiras através de concurso público, fazendo com que, no início da década de 1990, os vínculos celetistas ${ }^{2}$ no setor público fossem convertidos em vínculos estatutários.

Para compreender o processo de mudança que se impôs ao Estado a partir da Constituição de 1988, no sentido de reverter a lógica das políticas sociais até então seletivas e dirigidas para segmentos da população, há a necessidade de analisar a sua estrutura e as práticas de seus agentes institucionais. A Constituição de 1988 garantiu, enquanto um direito de todos e dever do Estado, o atendimento à saúde integral e universal, superando as limitações dos direitos tradicionais da legislação até então vigente. Essas conquistas do movimento da Reforma Sanitária Brasileira contribuíram para a superação da dicotomia histórica dos Direitos Trabalhista e Previdenciário, que atuavam como condutores hegemônicos das condições de vida e saúde no trabalho. A Lei 8080 de 1990 instituiu o Sistema Único de Saúde sob um novo enfoque pautado na universalidade, integralidade, equidade e controle social.

No relatório da primeira Conferência de Saúde do Trabalhador, publicado em dezembro de 1986, é discutida a defesa de uma política de recursos humanos voltada para os trabalhadores da saúde, mas que se restringe apenas à formação e remuneração, não discutindo a relação saúde-trabalho referente aos ambientes de trabalho.

Com o novo cenário político pós-constituinte, aliado às manifestações e atuações dos órgãos internacionais (OIT, OMS) e às pressões dos movimentos sociais na direção da necessidade que o Brasil instituísse uma política pública direcionada para a saúde do trabalhador, em 1993 foi criada a Comissão Interministerial da Saúde do Trabalhador (CIST), e, no final deste mesmo ano, foi instituído o Grupo Executivo Interministerial em Saúde do Trabalhador (GEISAT). Inicia-se neste momento a construção, de forma participativa e interministerial, de uma proposta para normalizar, regular e monitorar as relações de trabalho e seu impacto na saúde do trabalhador, a qual vai culminar com a 
Política de Atenção ao Servidor Público (PASS), como pode ser acompanhado no quadro abaixo:

Quadro 1 - 0 Processo de Construção da Política de Atenção à Saúde do Servidor na Linha do Tempo

\begin{tabular}{l|l|l|l|l|l|l|l|}
1988 & 1990 & 1993 & 1993 & 2004 & 2006 & 2009 & 2012 \\
\hline CF & RJU & CIST & $\begin{array}{l}\text { GEI- } \\
\text { SAT }\end{array}$ & PNSST & SISOSP & SIASS & PASS
\end{tabular}$\rightarrow$

Para que esta política, que tem como princípios a integralidade, a intersetorialidade e a participação social, fosse construída foi necessário compatibilizar e integrar as ações de saúde e trabalho desenvolvidas, entre outros, pelos Ministérios do Trabalho, da Saúde, do Meio Ambiente e da Previdência e Assistência Social, de forma a coordenar e articular o atendimento das demandas referentes à saúde dos trabalhadores.

31 A segunda Conferência de Saúde do Trabalhador, realizada em 1994, já após a promulgação da Lei 8080 de 1990, aprova propostas sobre a regulamentação da formação de recursos humanos para a saúde, com ampliação de seu quadro de pessoal e implantação de Plano de Carreira, Cargos e Salários - (PCCS). Além disso, preocupa-se em garantir ações de vigilância e fiscalização nas instituições públicas e privadas e propõe a criação de Comissões de Saúde do Trabalhador nos serviços públicos e privados, destacando a exigência de que o serviço público passe a emitir a Comunicação de Acidente de Trabalho - (CAT). Recomenda que a Comissão de Saúde do servidor público seja amplamente discutida nas bases da categoria a nível federal, estadual e municipal, junto aos seus sindicatos e representações. Esta comissão seria composta exclusivamente por servidores, eleitos por seus pares (Brasil, 1994:20).

Este processo de construção de uma política integral que articulasse saúde, trabalho e ambiente envolveu uma acirrada disputa entre necessidades de saúde, escolhas da comunidade, decisões políticas e recursos disponíveis.

Embora os Grupos de Trabalho tenham evoluído no sentido da aprovação de Normas Regulamentadoras de: Segurança e Saúde para os trabalhadores do serviço público; um Programa de Prevenção de Riscos Ambientais; e um

Programa de Controle Médico de Saúde Ocupacional, até o início dos anos 2000, as políticas públicas não tinham tido nenhuma atenção, organizada em nível federal, direcionada aos ambientes e processo de trabalho que garantissem a saúde integral ao servidor público.

\section{A Política Nacional de Segurança e Saúde do Trabalhador (PNSST) e o Sistema de Atenção á Saúde do Servidor Público (SIASS)}

35 As Políticas de Saúde, como as demais políticas públicas refletem os projetos de governo e a correlação de forças políticas que ocupa o poder. Em 2003, assume a Presidência do governo brasileiro Luis Inácio Lula da Silva, com a proposta de revitalização do Estado, que deveria ter um papel ativo na redução das desigualdades e na promoção do desenvolvimento. ${ }^{3}$ De acordo com Teixeira (2012):

Nesse momento ganha força no interior do governo a adoção de medidas orientadas à reversão do quadro de precarização do trabalho. Tais medidas foram ancoradas na 
ideia de que parte da solução para o desafio de aumentar a formalização do mercado de trabalho no país estaria no desenvolvimento econômico (Teixeira, 2012:204). Trabalhador (PNSST), na qual são estabelecidos os princípios a serem seguidos no sentido de superar a fragmentação, desarticulação e superposição das ações implementadas pelos setores trabalho, previdência social, saúde e meio ambiente.

42 A PNSST, em sua proposta, qualifica o trabalho como determinante social de saúde da população e, assim, em suas diretrizes, a Saúde do Trabalhador é colocada como uma responsabilidade ampla e coletiva do Estado no sentido de garantir o direito pleno a "todos os homens e mulheres que exercem atividades para sustento próprio e/ou de seus dependentes, qualquer que seja sua forma de inserção no mercado de trabalho, no setor formal ou informal da economia" (PNSST, 2004:04), sendo, portanto, uma política universal e inclusiva.

O documento da PNSST define diretrizes, responsabilidades institucionais e mecanismos de financiamento, gestão, acompanhamento e controle social, com o objetivo de orientar os planos de trabalho e ações intra e intersectoriais no âmbito da saúde do trabalhador. 
Entre as diretrizes desta política destaca-se a orientação para que o Estado cumpra seu papel de empregador, garantindo o atendimento de ações de segurança e saúde do trabalhador.

(...) para que o Estado cumpra seu papel na garantia dos direitos básicos de cidadania é necessário que a formulação e implementação das políticas e ações de governo sejam norteadas por abordagens transversais e intersetoriais. Nessa perspetiva, as ações de segurança e saúde do trabalhador exigem uma atuação multiprofissional, interdisciplinar e intersetorial capaz de contemplar a complexidade das relações produção-consumo-ambiente e saúde. (PNSST, 2004-03)

Para o cumprimento desta determinação legal foi criada, no âmbito da administração pública, uma unidade responsável pela construção de uma política voltada especificamente para as relações de trabalho-saúde do servidor público. Com esse objetivo, em 2006 foi instituído o Sistema Integrado de Saúde Ocupacional do Servidor Público Federal (SISOSP), através do Decreto n. ${ }^{\circ} .961$ de 13/11/2006, da Presidência da República. Desta forma, o Estado assume formalmente sua responsabilidade como empregador no âmbito da saúde ocupacional, uniformizando protocolos e procedimentos.

Esta iniciativa do Ministério do Planejamento foi o primeiro movimento da administração pública federal em direção ao processo de transformação no tratamento da atenção à saúde do servidor, tendo como objeto a relação saúdetrabalho.

Destaca-se que a política de saúde do trabalhador no setor público é estabelecida tardiamente e dissociada das ações voltadas aos trabalhadores celetistas, onde os serviços de saúde e segurança do trabalho são de responsabilidade dos próprios empregadores e apenas regulados e fiscalizados pelo Estado por parte do Ministério do Trabalho e Emprego.

48 No âmbito do setor público federal a estratégia de trabalho participativa desde, o princípio foi a formação de Grupos de Trabalhos nas instituições, composto por trabalhadores que já atuavam na área de atenção à saúde dos trabalhadores, para pensar e discutir a construção deste projeto de forma coletiva e participante.

Iniciado o processo de implementação do SISOSP, uma das primeiras iniciativas dos grupos de trabalho criados para este fim foi, em 2009, mudar o nome de Sistema Integrado de Saúde Ocupacional do Servidor Público Federal para Subsistema Integrado de Atenção à Saúde do Servidor (SIASS) ${ }^{4}$. A alteração do nome evidencia uma mudança de referencial teórico em relação à proposta inicial, no sentido da integralidade do conceito de Saúde do Trabalhador, uma vez que SISOSP demonstrava o alinhamento com a conceção restrita de saúde ocupacional.

O projeto para construção do SIASS previa que o Ministério do Planejamento fizesse a intermediação de convênios para organização de uma rede com unidades hierarquizadas, viabilizando sua operacionalização para atendimento em todo o território nacional.

O modelo de gestão em rede tinha como objetivo garantir mecanismos de controle, resolução de conflitos e tomada de decisão, necessários para dar o caráter de eficiência e efetividade a esta área de Atenção à Saúde do Servidor, contribuindo para a promoção social e para uma política de cidadania. 0 modelo de rede constitui-se como um desenho facilitador de desenvolvimento social importante no movimento de transformação do nível de saúde, tanto coletiva, quanto individual (Junqueira, 2000). Só através dele será possível atender as ações de saúde do servidor com resolutividade, integralidade e equidade. 
52 A metodologia de trabalho adotada incluiu a organização de encontros a nível nacional, com oficinas temáticas sobre a saúde do trabalhador, o que se constituiu não apenas como uma oportunidade de construir coletivamente o novo modelo, como também, como um espaço de difusão das informações e de diálogo. Além disso, a divulgação do trabalho que os grupos já organizados nos Estados vinham realizando não só incentivou outros grupos e unidades da federação a se engajarem no processo, como também promoveu uma reflexão sobre trabalho e saúde, que muito contribuiu para o processo.

O projeto de construção do SIASS previa que o Ministério do Planejamento fizesse a intermediação de convênios para organização das estruturas do Sistema, chamadas de Unidades de Referências, viabilizando sua operacionalização em todo o território nacional. A estratégia concebida para isto foi a pactuação entre os diferentes atores na definição dos papéis e responsabilidades de todos os envolvidos. Assim, coube ao Ministério do Planejamento a gestão da política; à Coordenação local do SIASS o papel de colaborador na gestão e às instituições federais selecionadas abrigar as Unidades de Referência para a operacionalização das ações mediante convênios onde devem ser alocados recursos financeiros e humanos.

Todo o processo de construção das bases legais tem sido um movimento coletivo desde a contribuição e ação dos grupos locais com reuniões periódicas como encontros nacionais.

O último passo do governo no sentido da institucionalização da Atenção à Saúde do Servidor Público foi o lançamento em 23/10/2012 da Política de Atenção à Saúde do Servidor Público (PASS) que está formulada e encaminhada, aguardando a assinatura da Presidenta da República.

Elaborado à luz das diretrizes da PNSST, o projeto estabelece como princípios fundamentais: (i) a gestão das informações epidemiológicas, de modo a conhecer o perfil de morbidade e mortalidade do servidor, especialmente aquele relacionado ao trabalho; (ii) a vigilância dos ambientes e processos de trabalho, de modo a conhecer as determinações primordiais dos problemas de saúde; (iii) a assistência requerida para viabilizar a melhor solução para os agravos que não puderem ser, até então, evitados; (iv) e a qualificação da perícia necessária para a resolução dos problemas decorrentes de um equacionamento ainda tateante em matéria de verdadeira promoção da saúde e prevenção dos danos. Essas abordagens devem ser interarticuladas, de modo interdisciplinar e intersetorial, melhor qualificando seu reconhecimento e a sua resolutividade (MPOG-SIASS, 2012).

\section{Considerações Finais}

O processo de construção e institucionalização da Política de Segurança e Saúde do Trabalhador no Brasil, na forma aqui apresentada, parte do pressuposto de que as políticas extrapolam o círculo governamental e não possuem um ciclo definido de nascimento e morte, mas se sucedem, se modificam e se complementam (Viana e Batista, 2009:73).

59 O caminho percorrido pela PNSST evidencia a forma com que o problema vem sendo tratado ao longo do tempo, em diferentes conjunturas políticas, nacionais e globais, o nível de organização da sociedade, os espaços de luta política e os modelos de saúde que conformam as propostas.

60 O princípio da saúde integral do trabalhador invoca o direito à saúde no seu sentido irrestrito da cidadania plena. Na prática, se traduz em ações que incluem a promoção, a 
prevenção e a assistência, a serem executadas de forma integrada e sempre através de uma abordagem interdisciplinar e intersetorial.

61 Pressupõe, assim, uma ação de articulação com ampla e efetiva participação de trabalhadores, de técnicos e pesquisadores, de instituições de ensino, de representantes sindicais, de serviços, da sociedade civil organizada e de outros atores institucionais e sociais. Para tanto é necessário que seja forjada uma aliança tácita de forma a se obter do Estado uma política coerente com as reais demandas.

Em termos de apontarmos para uma avaliação de cobertura (Graça 2000), a etapa atual do SIASS é de implantação da PASS que se fundamenta na perspetiva de universalização dos sistemas de saúde em que a atenção integral a saúde do trabalhador deve estar inserida. Segundo informações recentes em 2012 o SIASS contava com 1.671 profissionais com uma cobertura de 1.160 mil trabalhadores públicos federais representando cerca de $1,5 \%$ da População Economicamente Ativa. (Brasil-MPOG)

(aca-se que, se não representa um grande quantitativo de cobertura, tal é contudo um exemplo e uma tendência de forma de organização e gestão participativa para o restante do setor público sob a gestão dos Estados e municípios, poderes legislativo e judiciário e mesmo para setores empresariais.

A participação do trabalhador no processo de construção de políticas é o que legitima sua eficácia. Este ator detém o conhecimento ou o saber do processo de trabalho e do impacto do trabalho em sua saúde e, portanto, em sua vida.

Assim, a subjetividade, o ambiente e o processo de trabalho são importantes componentes para a relação empregador-empregado na atenção à saúde.

perspetiva deve ser destacada na construção da Política Nacional de Segurança e Saúde do Trabalhador (PNSST) e na Política de Atenção à Saúde do Servidor Público (PASS) o seu caráter inovador e transformador.

no processo de construção destas políticas a decisão política de construir-se o sistema e a sua base legal de forma participativa, compartilhada e universal, buscando (re)significar a relação trabalho, saúde e ambiente a partir da implantação de um modelo de atenção, fundamentado na integralidade das ações e na participação e controle social.

analisar as iniciativas do Estado direcionadas a estruturar a política de saúde do trabalhador no serviço público federal, ou seja, as estratégias de implementação da PNSST e da PASS, Andrade (2009: 91-92) identifica como fatores limitantes para a implementação deste modelo a estrutura hierarquizada e racionalizadora das instituições públicas e a dificuldade de que os trabalhadores tomem para si a corresponsabilidade na construção de um modelo de promoção da sua saúde e das suas condições de trabalho.

O acompanhamento do processo de institucionalização do SIASS mostra que há um avanço em relação à consolidação do novo modelo, mas a participação das associações e sindicatos ainda está se dando de forma retraída em relação à proposta de que o mesmo atue como protagonista na condução do processo de gestão, acompanhamento e controle social de sua saúde.

É importante chamar atenção para o fato de que este momento de implementação da Política se caracteriza como o tempo da negociação e da consolidação dos pactos estabelecidos. Para Viana e Batista, é na formulação que se estabelecem os grandes consensos, e ali tudo é possível; mas é no momento da implementação que se evidenciam 
os apoios, os interesses que concorrem, os grupos que disputam a hegemonia, enfim, a real potencialidade da política (Viana e Batista, 2009:78).

Neste artigo procurou-se recuperar, a partir dos documentos oficiais, a trajetória de formulação da Política de Saúde do Trabalhador, que tem como marco a "Constituição Cidadã" de 1988, e como objetivo reverter a lógica das políticas sociais até então seletivas e dirigidas para segmentos da população e que, a partir deste momento, passam a ter um caráter universal, de integralidade, equidade e hierarquizado.

A situação atual aponta para um avanço na direção da possibilidade da política que vem sendo implementada, contribuindo para a melhoria da qualidade de vida dos trabalhadores através de um Sistema de Saúde do Trabalhador pautado na relação saúde trabalho - ambiente, de base universal e com participação social.

Tomando como referência o método de análise de políticas públicas do ciclo de políticas (Viana e Batista, 2009:73), no caso da PNSST e da PASS, para que possa ser feita uma análise do processo de implementação, será necessário acompanhar como se vão estruturar os apoios, os recursos alocados para este fim, a disposição dos trabalhadores em participar, sua mobilização social e a abertura institucional para estruturação de um modelo organizacional democrático e flexível que possibilite a efetivação dos princípios que as orientam.

\section{BIBLIOGRAFIA}

ABRASCO (Associação Brasileira de Pós-Graduação em Saúde Coletiva), Rio de Janeiro, v. 10, n.o 4: 987-992.

ALEXANDER, J. (1998), “The ideological construction of risk: an analysis of corporate health promotion programs in the 1980's" Social Science \& Medicine, 26, 5: 559-567.

ANDRADE, E.T. (2009), O Processo de Implementação da Política de Atenção à Saúde do Trabalhador em Instituições Públicas Federais: o desafio da integralidade, Dissertação de Mestrado, Escola Nacional de Saúde Pública - Fiocruz, Rio de Janeiro: 12-49.

BRASIL. MPOG. (s.d), Boletim Estatístico Anual, n. 182. [Online] Disponível em: http://www.servidor .gov.br [Consultado em 18 de março de 2012].

CONRAD, P. e WALSH, D.C.(1992), “The new corporate health ethic: lifestyle and the social control of the work", International Journal of health Services, 22: 89-111.

DEDECCA, C.S.e (2006), "Flexibilidade e Regulação de um mercado de trabalho precário: a experiência brasileira", in Colóquio Internacional Novas Formas do Trabalho e do Desemprego: Brasil, Japão e França, numa perspetiva comparada; Departamento de Sociologia, São Paulo/USP; 2006. [Online] Disponível em: http:/www.centrodametropole.org.br, [consultado em maio de 2007]. DIAS, E. (2002), Aspetos Conceituais da Assistência à Saúde do Trabalhador. Anais da $2^{\underline{a}}$ Conferência Nacional da Saúde do Trabalhador - 1994, Brasília: DFS. 
FLEURY, S e OUVERNEY, A. (2009), "Política de Saúde: uma Política Social”, in L. Giovanella, S. Escorel, L. Lobato, J. Noronha e A. Carvalho, Políticas e Sistemas de Saúde no Brasil, Rio de Janeiro: Editora Fiocruz: 23-64.

GOMES, F.(2006) "Conflito Social e Welfare State: Estado e desenvolvimento social no Brasil”. Revista de Administração Pública - Fundação Getúlio Vargas, Rio de Janeiro, n.ำ 40: 201-233.

GRAÇA, L. (2000), Serviço de Saúde e Segurança no Trabalho em Portugal e na União Europeia: problemas e perspetivas, Lisboa, Universidade Nova de Lisboa, Escola Nacional de Saúde Pública; disciplina de Psicossociologia do Trabalho e das Organizações de Saúde. texto policopiado. [Online] Disponível em: http://www.ensp.unl.pt/lgraca/textos108.html. [Consultado em 18 de março de 2012].

JUNQUEIRA, Luciano (2000), “Intersetorialidade, Transetorialidade e Redes Sociais”, Revista de Administração Pública - Fundação Getúlio Vargas, Rio de Janeiro, v. 34, n.․ㅡ Especial: 35-45.

LACAZ, F (2007), "O Campo da Saúde do Trabalhador: resgatando conhecimentos e práticas sobre relações de trabalho”. Caderno de Saúde Pública, Rio de Janeiro, v. 23, n.ำ 4: 757-766.

LAURELL, A. e NORIEGA, M. (1989), "Para o Estudo de Saúde na sua Relação com o Processo de Produção", in Asa Laurell e Mariano Noriega, Processo de Produção e Saúde: trabalho e desgaste operário, São Paulo, SP: Hucitec.

MACHADO, J.H. (2005), “Ação transversal intersetorial - entre o racional e o conjuntural”, in Encontro Preparatório para a $3^{a}$ Conferência Nacional de Saúde do Trabalhador, Brasília: DF.

MACHADO, J.H. (2005), “A propósito da Vigilância em Saúde do Trabalhador”, Ciência \&Saúde Coletiva.

ODDONE, I. et al. (1986), “Conquistas para Modifi cação do Ambiente de Trabalho”, in I. Oddone et al., Ambiente de Trabalho: A Luta dos Trabalhadores pela Saúde, São Paulo, SP: Hucitec.

POLÍTICA NACIONAL DE SEGURANÇA E SAÚDE DO TRABALHADOR (2004), Versão pronta após sugestões incluídas sugestões do Seminário Preparatório, dos AFTs e das DRTs - Brasília, DF. [Online] Disponível em: http://portal.saude.gov.br/portal/arquivos/pdf/ insumos_portaria_interministerial_800.pdf [consultado em outubro de 2008].

TAMBELLINI, A.M. (1986), “Avanços na formulação de uma Política Nacional de Saúde no Brasil: As atividades subordinadas à área das relações produção e saúde", Conferência Nacional de Saúde dos Trabalhadores, Brasília.

TEIXEIRA, M. e GONDIM, R. (2012), "Mudanças nas Políticas de Trabalho e da educação (197-225)” in C. Machado, T. Batista, L. Lima, Políticas de Saúde no Brasil-continuidades e mudanças, Rio de Janeiro: Editora Fiocruz.

VASCONCELLOS, L.C.F e OLIVEIRA, M.H. (2000), “As Políticas Públicas Brasileiras de Saúde do Trabalhador: Tempos de Avaliação”, Saúde em Debate, Rio de Janeiro, v. 24, 55: 92-103.

VIANA, A.L. e BATISTA, T. (2009), in L. Giovanella, S. Escorel, L. Lobato, J. Noronha e A. Carvalho, Políticas e Sistemas de Saúde no Brasil, Rio de Janeiro, Editora Fiocruz: 65-106.

\section{NOTAS}

2. Vínculos celetistas - forma de relação de emprego estabelecido pela Consolidação das Leis do Trabalho CLT.

3. Documento Gestão Pública para um Brasil de Todos (Brasil/MPOG/Seges, 2003)

4. Decreto $n^{\circ} 6.833$, de 29 de abril de 2009 . 
1. Os trabalhadores do governo que pertencem ao quadro próprio do Estado são chamados de servidores públicos.

\section{RESUMOS}

Este artigo realiza um ensaio crítico sobre o processo de formulação da Política de Saúde para os trabalhadores do setor público no Brasil utilizando como referência os conceitos de políticas sociais e de saúde, (Fleury, 2009), saúde do trabalhador, (Oddone, 1986), regulação do trabalho, (Dedecca, 2006) e avaliação de políticas de saúde, (Viana e Batista, 2009). Partindo desta base conceitual são apresentados e discutidos os documentos oficiais e a legislação que institui a Política Nacional de Segurança e Saúde do Trabalhador (PNSST) e a Política de Atenção à Saúde dos Servidores públicos ${ }^{1}$ (PASS). Os resultados desta discussão corroboram a tese de que a formulação e implementação de políticas públicas é um espaço de luta política e ideológica, com reflexo nas agendas públicas e na relação do Estado com a sociedade.

This article presents a critical essay on the formulation process of Health Policy for public setor workers in Brazil based on the concepts of social and health policies (Fleury, 2009), Worker's Health, (Oddone, 1986 Tambelline, 1985, 1986), work regulation (Dedecca, 2006) and evaluation of health policies (Avila and Batista 2009).Taking into consideration such conceptual basis official documents and legislation establishing the National Policy on Worker's Health and Safety (PNSST) and Politics of Health Care Civil Servants (PASS) are presented and discussed. The results of this discussion corroborate the thesis that the formulation and implementation of public policies is an area of political and ideological struggle, with impacts on public agendas and in the relationship between the state and society.

Cet article présente un essai critique sur le processus de formulation de la politique de santé pour les travailleurs du secteur public au Brésil em utilisant comme référence les concepts de politiques sociales et de santé, Fleury (2009), la santé du travailleur, Oddone (1986) Tambelline, 1985, 1986, la régulation du travaille, Dedecca (2006) et l'évaluation des politiques de santé, Avila et Batista (2009). En s'appuyant sur ce concepts ont présentés et discutés les documents officiels et les lois instituant de la Politique Nationale de Sécurité et Santé au Travail (PNSST) et la Politique d'Attention à la Santé des Serveurs publique (PASS). Les résultats de cette discussion corroborent la thèse selon laquelle la formulation et la mise en œuvre des politiques publiques est un domaine de la lutte politique et idéologique, avec des impacts sur les agendas publics et la relation entre l'État et la société.

\section{ÍNDICE}

Mots-clés: politique de santé, travailleur de la santé, le serviteur de la santé publique Palavras-chave: saúde do trabalhador, política de saúde, saúde e trabalho, saúde do servidor público

Keywords: worker's health, health policy, healthand work, health of public servant 


\section{AUTORES}

\section{ELSA THOMÉ DE ANDRADE}

Analista de C\&T da Escola Nacional de Saúde Pública Sergio Arouca ENSP/FIOCRUZ, Mestre em Saúde Pública

migmau@terra.com.br

\section{MARIA INÊS CARSALADE MARTINS}

Pesquisadora da Escola Nacional de Saúde Pública Sergio Arouca ENSP/FIOCRUZ, Doutora em Saúde Coletiva

mines@ensp.fiocruz.br

\section{JORGE HUET MACHADO}

Pesquisador da Escola Nacional de Saúde Pública Sergio Arouca ENSP/FIOCRUZ, Doutor em Saúde Coletiva

jorgemesquita@yahoo.com.br 